\title{
Liderazgo en docentes de básica primaria de una institución pública de la ciudad de Bucaramanga, Santander, Colombia
}

\section{Leadership in primary school teachers of a public institution in the city of Bucaramanga, Santander, Colombia}

\author{
Mónica Alexandra Ayala Mejía \\ Licenciada en Educación Preescolar \\ Universidad Autónoma de Bucaramanga, Santander, Colombia \\ Correo electrónico: mayala3@unab.edu.co
}

Recibido: 17 de julio de 2017. Aprobado: 3 de septiembre de 2017

\section{Cómo citar este artículo}

Ayala, M.A. (2018). Liderazgo en docentes de básica primaria de una institución pública de la ciudad de Bucaramanga, Santander, Colombia. Espiral, Revista de Docencia e Investigación, 8(1), 53 - 64.

\section{Resumen}

Objetivo. El liderazgo educativo se ha consolidado como un elemento fundamental al momento de fortalecer la calidad en la educación. Sin embargo, el enfoque que se le ha dado en las instituciones educativas lo sitúa en el ámbito administrativo sin involucrar a la comunidad. Nos proponemos determinar en este artículo, la presencia del liderazgo distribuido en el trabajo docente de una institución básica primaria de la ciudad de Bucaramanga.

Metodología. Para ello, se aplicaron instrumentos como cuestionarios y entrevistas, los cuales fueron insumo de información, al igual que el proceso de observación realizado mediante una lista de chequeo aplicada por el investigador, los cuales permitieron conocer las percepciones que tenían los docentes y el coordinador sobre el concepto de líder, el liderazgo y los elementos del mismo que se presentaban en la institución.

Resultados. En esta dirección se establecieron cinco categorías y veintiocho subcategorías básicas para realizar el proceso de análisis y triangulación de la información, que en la institución no se presenta un liderazgo distribuido debido a que los docentes no perciben claramente qué tipo de liderazgo se ejerce en la organización y los lineamientos que orientan esta labor son atribuidos a la coordinación la cual presenta un liderazgo basado en la subordinación a los seguidores.

Palabras claves: Liderazgo, liderazgo educativo, tipos de liderazgo, liderazgo distribuido, docentes.

\begin{abstract}
Objective. Educational leadership has been consolidated as a fundamental element at the time to strengthen the quality of education. However, the approach that has been given in educational institutions, is located in the administrative field without involving the community. We intend to determine in this article, the presence of leadership distributed in the teaching work of a primary basic institution in the city of Bucaramanga.
\end{abstract}

Methodology. For this, instruments such as questionnaires and interviews were applied, which were the information input, as well as the observation process carried out by a checklist applied by the researcher, which allowed to know the perceptions that the teachers and the coordinator had about the concept of leader, leadership and the elements of leadership that were presented in the institution.

Results. In this direction, five categories and twentyeight basic subcategories were established to carry out the process of analysis and triangulation of information. The institution does not present a distributed leadership because the teachers do not perceive clearly what type of leadership is exercised in the organization and the guidelines that guide this work are attributed to the coordination which presents a leadership based on the subordination of the member of the teachers.

Keywords: Leadership, educational leadership, types of leadership, distributed leadership, teachers. 


\section{Introducción}

A partir de la inquietud generada por la ausencia de transformaciones significativas en el sistema educativo colombiano y el papel que juegan los docentes en la educación como líderes orientadores de los procesos de formación, surgió la necesidad de determinar la presencia del liderazgo distribuido en el trabajo docente de una institución de educación básica primaria de la ciudad de Bucaramanga.

A partir de lo anterior se organizó la propuesta de investigación apoyada en un paradigma cualitativo, enfocado en un diseño metodológico basado en la teoría fundamentada y se empleó como instrumentos para la recolección de información la entrevista, la encuesta y una lista de chequeo.

Este artículo está conformado por tres secciones. En la primera se presenta la metodología desarrollada en la investigación; la segun$\mathrm{da}$, los resultados obtenidos; y finalmente, las conclusiones.

\section{Metodología}

La investigación se basó en el paradigma cualitativo, que toma la recolección y el análisis de datos como medio para evaluar el desarrollo natural de un contexto particular, es así, como esta metodología, busca generar nuevas teorías basadas en las representaciones dadas por los individuos participantes en la investigación (Hernández, Fernández \& Baptista, 2014), además centra su interés en "la lectura que la gente hace de su realidad" (Giroux \& Tremblay, 2009, p. 39).

El objetivo de la investigación fue determinar la presencia del liderazgo distribuido en el trabajo docente de una institución de educación básica primaria de la ciudad de Bucaramanga. El estudio se basó en el análisis de la percepción que tienen los docentes y el coordinador sobre el tema y las implicaciones que tiene en el desarrollo de los procesos educativos que se llevan a cabo en la institución.

\section{Método de investigación}

Esta investigación es de tipo descriptiva, la cual según Hernández, Fernández y Baptista (2014) se caracteriza por analizar y explicar los rasgos, propiedades o cualidades de una situación en particular, por medio de la recolección de datos referentes al fenómeno de investigación, estableciendo una serie de temas sobre los cuales puntualizar. Con la presente investigación se buscó determinar la presencia del liderazgo distribuido en el trabajo docente de una institución de educación básica primaria de la ciudad de Bucaramanga, bajo el enfoque cualitativo que "se fundamenta en una perspectiva interpretativa centrada en el entendimiento del significado de las acciones de los seres vivos, sobre todo de los humanos y sus instituciones" (2014, p. 9).

Es por esta razón, que la investigación toma la temática del liderazgo distribuido como fuente de estudio en la que intervienen la relación entre las personas, en este caso, el coordinador y docentes de la institución educativa para describir la percepción que tienen sobre este fenómeno.

\section{Diseño metodológico}

El diseño cualitativo se enmarcó en la teoría fundamentada, en la que "el investigador produce una explicación general o teoría respecto a un fenómeno, proceso, acción o interacciones que se aplican a un contexto concreto y desde la perspectiva de diversos participantes" (Hernández, Fernández y Baptista, 2014, p. 472). Esta teoría basa su postura en que los fundamentos teóricos emergen de los datos recolectados a lo largo de la investigación y es de esta forma que se da la comprensión de un fenómeno, para nuestro caso resultó de oportuno beneficio por cuanto se trata del fortalecimiento de los procesos educativos a través de los actos innovadores de los maestros.

Los autores Glaser y Strauss (1967) citados por Hernández, Fernández y Baptista (2014) proponen dos diseños de teoría fundamenta- 


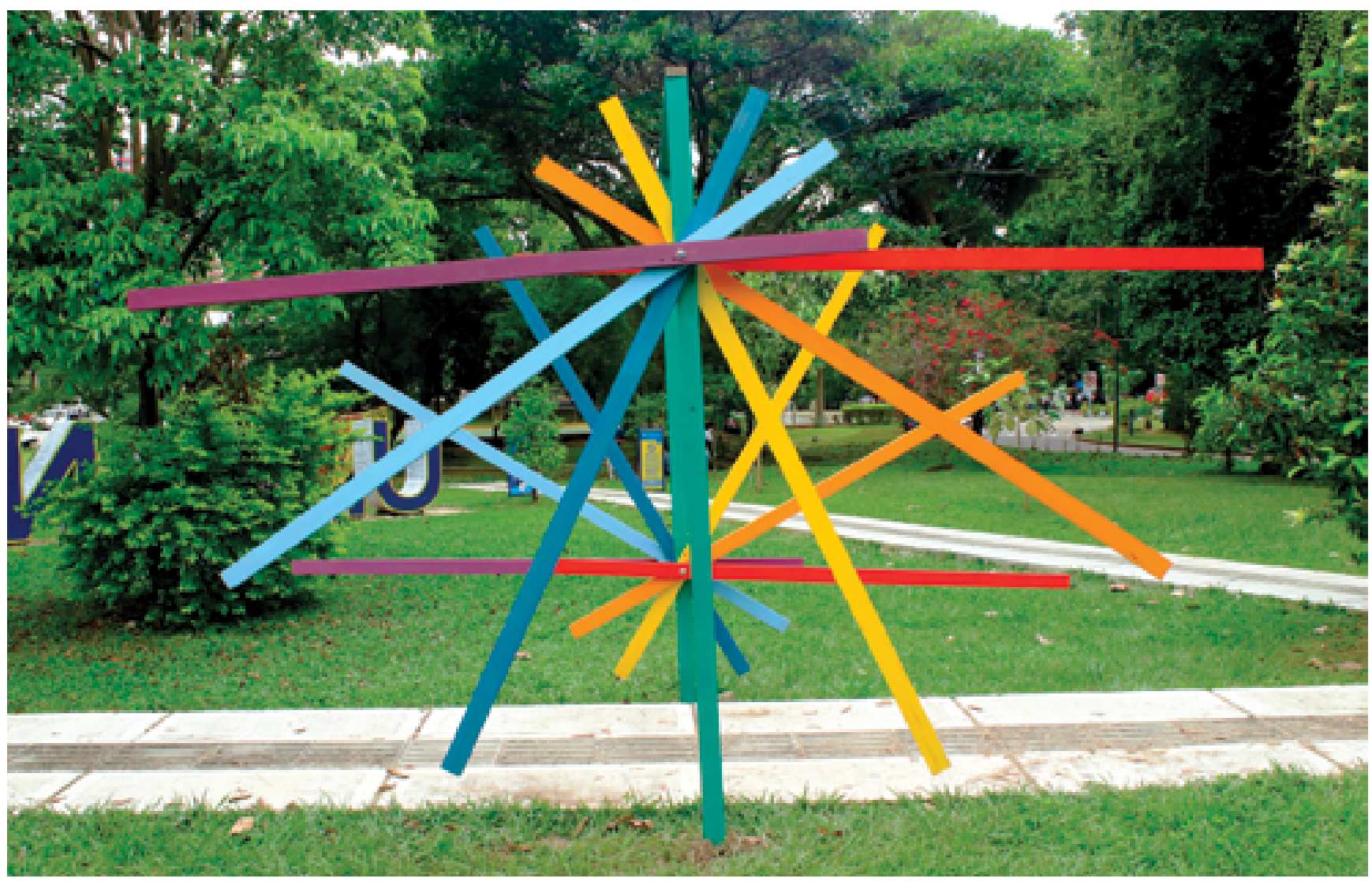

Proyecto Movimiento Cromático.

da, el diseño emergente y el sistemático. Para los análisis de la investigación se tomó el diseño sistemático, este tipo de diseño hace el siguiente proceso en el análisis de los datos: recolección de datos, codificación abierta, codificación axial, codificación selectiva y visualización de la teoría.

Al desarrollar el proceso de codificación abierta del que emergen las distintas categorías, se elige la categoría de mayor relevancia a la que se identificará como categoría central y estará acompañada del objetivo de investigación para posteriormente relacionarla con las demás categorías y llegar a la codificación axial, donde se construye el modelo denominado paradigma codificado.

En la codificación selectiva se identifican los conceptos, acciones e interacciones involucrados, para forjar un esquema y fundamentarlo a través de la comparación de los diferentes componentes y de esta forma generar hipótesis que permitan relacionar las categorías y describir el fenómeno.

Para el caso particular de la investigación, se buscó establecer la presencia del liderazgo distribuido en una institución básica primaria, que se desarrolló a partir de las percepciones dadas por los participantes, las cuales fueron insumo de la codificación y categorización que permitió hallar cinco categorías centrales y las subsidiarias.

Para llevar a cabo este diseño metodológico se estructuró un árbol de categorías y subcategorías tomando como base el marco teórico y se definieron y describieron las categorías y subcategorías previas y emergentes que soportaron el análisis de los datos.

\section{Población, participantes y selección de la muestra}

El tipo de muestra que se tomó en función de obtener los datos necesarios, fue por con- 
veniencia, la cual se caracteriza por realizar una elección a través de procedimientos no aleatorios de una muestra que posea características relacionadas a las de la población objetivo (Casal \& Mateu, 2003). Estas muestras se caracterizan por tomar casos a los que el investigador puede acceder (Hernández, Fernández \& Baptista, 2014).

La muestra de esta investigación estuvo integrada por 11 docentes y el coordinador de una institución educativa pública de la ciudad de Bucaramanga, quienes aprobaron su participación a través de la firma del consentimiento informado.

\section{Instrumentos}

Para esta investigación cualitativa se utilizaron tres instrumentos con el fin de obtener los datos necesarios para responder la pregunta de investigación. La recolección de datos se llevó a cabo en el ambiente escolar y la cotidianidad de la vida laboral de los docentes, sus experiencias, pensamientos y vivencias que fueron expresadas al investigador.

Entrevista. Se diseñó una entrevista estructurada, la cual se aplicó a los docentes y al coordinador de la institución, basada en una serie de preguntas predeterminadas y validadas por jueces expertos.

Cuestionario. Se aplicó a los docentes un cuestionario adaptado de la propuesta de (Celis \& Sánchez, 2012) con preguntas abiertas y validadas por jueces expertos.

Lista de chequeo. Se utilizó la lista de chequeo como método de observación y verificación en tres momentos de reunión entre docentes y el coordinador.

\section{Resultados}

La información obtenida fue insumo de la codificación y la categorización, siguiendo el diseño sistemático de la teoría fundamentada.
De acuerdo con la metodología, las categorías surgieron luego de aplicar los instrumentos, el proceso de codificación y la identificación de interacciones entre sí. Este proceso contó con el uso del software QRS NVIVO2 que permitió hallar 5 categorías y 28 subcategorías, para dar respuesta a la pregunta de investigación planteada.

Los resultados que se presentan a continuación, permitieron conocer una realidad específica compartida por la población objeto de estudio y la caracterización del liderazgo que se desarrollaba en la organización. La presentación de los resultados y análisis de los datos se van a exponer teniendo en cuenta el árbol de categorías presentado en la metodología y el diagrama de resultados.

Como punto de partida, se identificaron nueve tipologías del liderazgo entre las percepciones de los participantes, de las cuales seis comparten elementos característicos con el liderazgo distribuido; partiendo del liderazgo visionario, que se caracteriza por contar con líderes que orientan a los demás en una misma visión para lograr las metas planteadas (Robbins, 2004), este fue el que presentó mayor frecuencia, seguido por el liderazgo transformacional, liderazgo participativo, que son tipologías que se caracterizan por promover a través del liderazgo, la transformación de las organizaciones por medio de la participación del equipo de trabajo (Leithwood \& Duke, 1999). En este apartado, es importante resaltar que las características señaladas previamente hacen parte de los elementos que identifican el liderazgo distribuido, el cual toma características propias y de diversos estilos de liderazgo para lograr una tipología que se enmarque en el ámbito educativo.

Con una frecuencia intermedia, se presentaron el liderazgo autocrático y el liderazgo afiliativo en las apreciaciones de los participantes quienes consideraban que en las organizaciones se podían presentar líderes que imponen 
su voluntad, dando órdenes a los seguidores y coartando la expresión de estos (Bass \& Bass, 2008; Yulk, 2010) y líderes, que por lo contrario, dan gran importancia a las relaciones que se presentan entre el equipo (Goleman, 2005) siendo este, uno de los factores que también se observa en el desarrollo de un liderazgo distribuido, que es compartido con el liderazgo de tipo afiliativo.

El liderazgo de rasgos, liderazgo ejemplar, liderazgo democrático y el liderazgo distribuido presentaron una menor frecuencia. El primero hace referencia a líderes con cualidades innatas para ejercer la función de líder (Lupano \& Castro, 2005). El segundo caracteriza a líderes que son modelo para sus seguidores (Goleman, 2005). El tercero como lo indica su nombre, busca que la labor de liderar se lleve a cabo de una forma democrática, en la cual participen todos los miembros que hacen parte de la organización (Goleman, 2005), este tipo de liderazgo se acerca de forma muy significativa a la percepción que tiene el liderazgo distribuido, contando con una gran cantidad de elementos en común, entre ellos la participación en la toma de decisiones.

Por último, se manifestó el liderazgo distribuido, que se caracteriza por ser un liderazgo que se lleva a cabo en los ámbitos educativos, partiendo de la premisa que todas las personas que hacen parte de una organización pueden ejercer la labor de liderar, lo que conlleva que esta tarea permee a cada uno de sus miembros (Spillane, 2006).

Para el caso de esta investigación, las percepciones de los participantes derivaron en numerosas tipologías que cuentan con elementos presentes en el liderazgo distribuido, como el trabajo en equipo, basado en la colaboración, compromiso e intercambio de ideas; y la distribución de roles de forma coordinada, teniendo en cuenta las habilidades de los integrantes del grupo. Estos elementos aportan al desarrollo de un liderazgo significativo, relevante y efecti- vo en las organizaciones y pueden convertirse en el punto de partida para desarrollar un liderazgo de tipo distribuido en la institución.

Después de conocer la percepción de los participantes del concepto de liderazgo, se aplicaron preguntas, tanto en la entrevista como en el cuestionario, para conocer los elementos que caracterizan el liderazgo en la institución educativa, surgiendo los siguientes: trabajo en equipo, distribución de roles, toma de decisiones y desarrollo del liderazgo.

En el esquema se puede observar que, en cada ítem se realizó un paralelo entre los elementos del liderazgo presentes en la institución y los que caracterizan al liderazgo distribuido donde se subrayan las siguientes relaciones encontradas:

Trabajo en equipo. Este elemento se presentó en la institución educativa caracterizado en 10 subcategorías: La primera, es el intercambio de experiencias, conocimientos y emociones, en la que se evidenció que en el equipo de trabajo de la institución existía un alto interés en los docentes por compartir conocimientos, experiencias laborales y sentimientos, con el objetivo de favorecer y mejorar el crecimiento profesional y las prácticas que se realizaban en la institución. Esta subcategoría que se presentó en la muestra, se relaciona directamente con una de las características que se dan en el trabajo en equipo según el liderazgo distribuido y es el intercambio de conocimientos, en el cual prevalece el compartir de ideas que favorece la adquisición de nuevos aprendizajes, que permiten que se desarrolle un trabajo más eficaz en el que todos participen (Gronn, 2002).

Colaboración y compromiso es la segunda subcategoría que se presentó en la institución, que hace parte de los elementos que definen el trabajo en equipo en el liderazgo distribuido, con el nombre de colaboración, participación y compromiso. Esta subcategoría, muestra cómo el equipo de trabajo de la 
institución cuenta con un personal comprometido, que realiza acciones colaborativas encaminadas al logro de los objetivos propuestos, lo que refleja el desarrollo en la institución de esta particularidad del liderazgo distribuido (Spillane, 2006).

La ausencia de espacios para la reflexión se presentó como un aspecto para fortalecer en la institución, debido a que los docentes manifestaban que los momentos que se les brindaban para capacitarse y reflexionar sobre su labor eran escasos, lo que no permitía realizar una retroalimentación adecuada para fortalecer los procesos que se desarrollaban.

Otra de las subcategorías que caracterizaron el trabajo en equipo, fue el proceso de participación, el cual reflejó que en la institución se presentaba intercambio de opiniones, ideas y pensamientos por parte de los miembros, favoreciendo el trabajo en equipo. Esta característica hace parte de los elementos del trabajo en equipo del liderazgo distribuido, ya que según Spillane, Halverson y Diamond (2004) el liderazgo distribuido involucra a los sujetos para que aporten y participen del liderazgo, permitiendo que se desarrolle en forma compartida.

La falta de capacitación e innovación, es otro de los aspectos que según los participantes requerían un mayor trabajo, disposición de tiempo y espacios para desarrollar actividades que brindaran al equipo nuevas herramientas que fortalecieran su trabajo y que aportaran al crecimiento de la institución.

Mostrando frecuencias bajas, se presentó en el trabajo en equipo la interacción entre docentes y la comunicación entre docentes y el coordinador, los cuales eran factores que apuntaban al desarrollo de la comunicación en la institución para trabajar en consenso por el logro de las metas que se planteaban dentro de la misma. Estos aspectos apuntan a la comunicación asertiva que es un factor que caracteriza el trabajo en equipo en el liderazgo distribuido, ya que según Leithwood (1996) las organizaciones deben coordinar su equipo de trabajo, con el fin de llegar a acuerdos que permitan el alcance de las metas planteadas, por lo cual, los líderes encaminan a las personas a esa dirección a través de procesos de comunicación y participación.

Cerrando la caracterización del trabajo en equipo en la institución, se presentaron la falta de apoyo y la desconfianza, los cuales son aspectos que, de acuerdo con los participantes requerían una mayor reflexión por parte del equipo para que se presentaran.

Distribución de roles. Este segundo elemento presentó cuatro subcategorías que permitieron su caracterización en la institución.

La coordinación de labores, es la subcategoría que encabeza el elemento de distribución de roles donde los participantes reflejaron que los trabajos y las tareas que se realizaban en la institución se desarrollaban partiendo de una distribución de labores entre los docentes y el coordinador, permitiendo la organización y funcionamiento de la institución. Esta coordinación de labores se presenta como una de las características de la distribución de roles en el liderazgo distribuido. En esta tipología de liderazgo, este aspecto que se refiere a la coordinación entre todo el equipo de trabajo apunta a la idea principal del liderazgo distribuido, la cual es descentralizar la gestión educativa, brindando a todos los integrantes de la organización un rol de mayor importancia (Heck \& Hallinger, 2005).

La segunda subcategoría que se presenta para este apartado, es la claridad en el rol, en la que se reflejó en el equipo de trabajo participante del estudio, claridad en las funciones que desempeñaban $y$, además, que dichas funciones se delegaban teniendo en cuenta las habilidades y fortalezas con las que cada persona le podía aportar a la institución. Esta característica que se veía reflejada en la insti- 
tución, hace parte del liderazgo distribuido, el cual busca como medio para fortalecer el proceso del liderazgo, tener en cuenta las habilidades que poseen los miembros del equipo de trabajo, las cuales pueden favorecer y aportar a la organización como plantean López, Sánchez, y Altopiedi (2011) el liderazgo puede proceder de cualquier lugar dentro de la escuela, y este tiene que ver con las cualidades que desarrollan los grupos e individuos en un contexto particular.

La planificación, es otra de las características que se presentaba en cuanto a la distribución de roles en la institución. Los participantes manifestaron que contaban con una organización de su trabajo y ellos aportaban en la construcción de la misma, pero que esta podía ser elaborada de una mejor forma, para que se optimizaran los resultados, como se plantea en liderazgo de tipo distribuido, donde la planificación es una característica que se hace presente dirigida al diseño de planes, estrategias y acciones orientadas a la búsqueda de resultados efectivos para la organización.

La última subcategoría que surgió en cuanto a la distribución de roles con una frecuencia representativa baja, presentándose como un aspecto para fortalecer, fue la imposición de roles, en la que algunos participantes expresaron que gran parte de las tareas eran impuestas por la dirección a los docentes de la institución educativa, sin tener en cuenta su opinión, para su planeación y desarrollo.

Toma de decisiones. Este elemento presentó cuatro subcategorías que lo caracterizaron en la institución. La subordinación se presentó como la primera subcategoría que caracterizó la toma de decisiones en la institución, observándose en las manifestaciones de los participantes, la carencia de autonomía que tenían en cuanto a la toma de decisiones, debido a

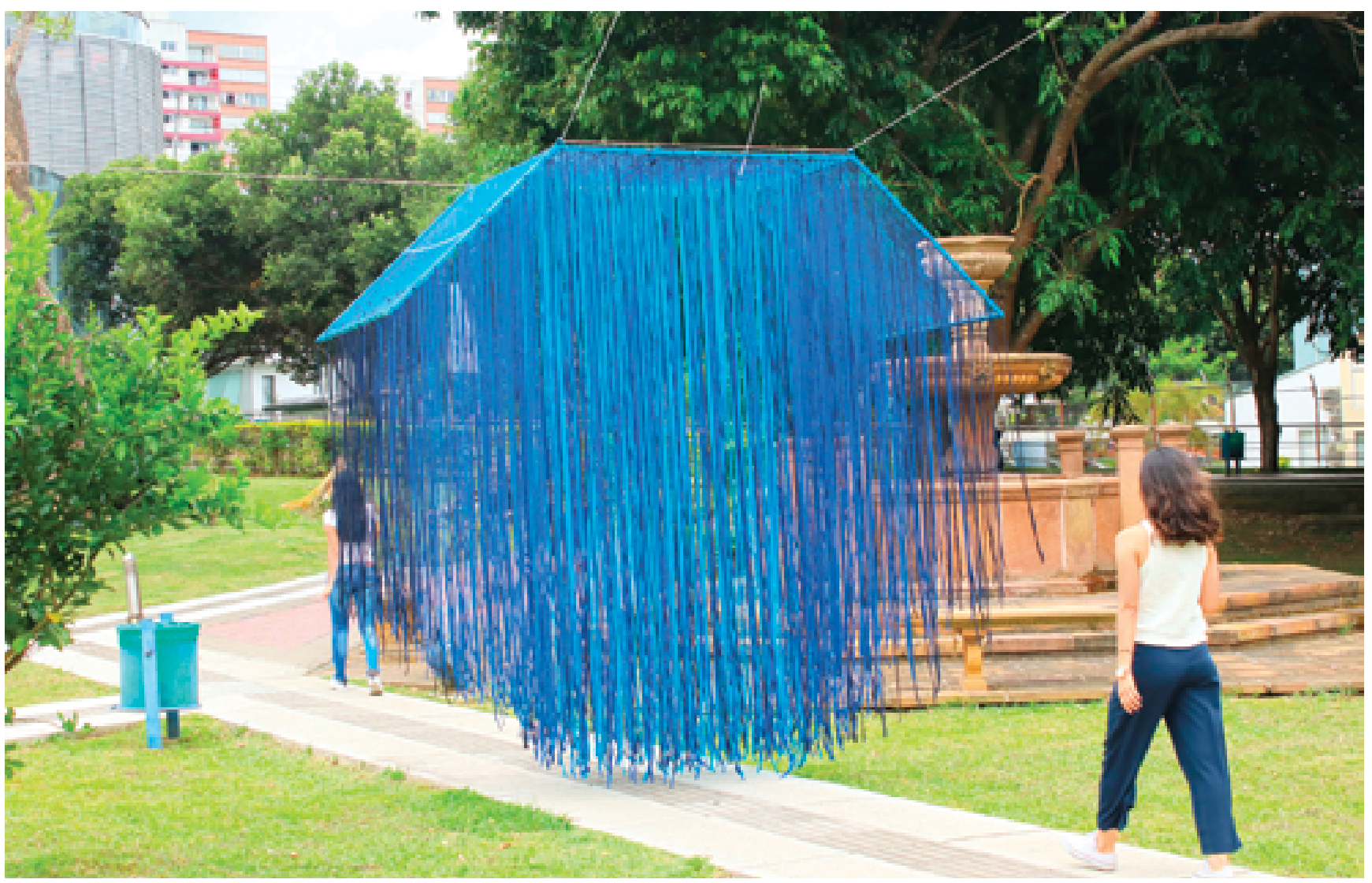

Proyecto Naciente. 
que se regían por lineamientos dados por la dirección, los cuales no tenía en cuenta las opiniones del equipo de trabajo, llevando a que la toma de decisiones, se realizara de forma autoritaria y subordinada, prevaleciendo la palabra de una persona. Este aspecto va en contravía con lo que plantea el liderazgo distribuido, en el cual la toma de decisiones se caracteriza por integrar a todas las personas que hacen parte de la organización.

La segunda subcategoría que caracterizó la toma de decisiones en la institución, fue la autonomía, enfocada a la libertad que tienen los docentes en su trabajo pedagógico en el aula, donde podían desarrollar las estrategias que creían pertinentes para el logro de los objetivos con sus estudiantes.

La democracia y participación, es otra de las subcategorías que se presentó, con una frecuencia menor, ya que los docentes participantes manifestaban que se les permitía participar y que en algunas ocasiones sus opiniones eran tenidas en cuenta en la toma de decisiones.

La democracia y participación en la toma de decisiones es un elemento que caracteriza la tipología del liderazgo distribuido, debido a que su fundamento está planteado en un liderazgo que logre permear a toda la organización desde todas las etapas que este conlleve, incluyendo la toma de decisiones. Para el liderazgo de tipo distribuido, las decisiones deben ser tomadas en consenso por todas las personas que hacen parte de la organización, debido a que estas son quienes conocen la comunidad y sus necesidades (Heck \& Hallinger, 2005).

La última subcategoría que se presentó en cuanto a la toma de decisiones en la institución, fue la búsqueda de soluciones. Esta se mostró con una frecuencia baja, dónde algunos participantes manifestaban la importancia de buscar estrategias que apuntaran a mejorar los procesos que se llevaban a cabo en la institución.
Esta subcategoría es una de las características de la toma de decisiones en las organizaciones cuando su liderazgo es de tipo distribuido, ya que este tipo de liderazgo valora las ideas del equipo de trabajo, teniendo presente que las soluciones a las situaciones que se presentan pueden surgir del equipo de trabajo y todo integrante que haga parte de la organización (Heck \& Hallinger, 2005). Desde esta mirada el liderazgo puede expandirse y ser ejercido por cada miembro que esté involucrado en la misma.

Desarrollo del liderazgo. En este elemento se presentaron dos subcategorías que hacen referencia a dos tipos de percepción en cuanto al desarrollo del liderazgo: la ausencia de liderazgo en los docentes y la promoción de liderazgo docentes, donde el primero mostró una mayor frecuencia en la institución.

La primera subcategoría que surgió gracias a las expresiones de los participantes, fue la ausencia de liderazgo en los docentes, debido a la no promoción del mismo por parte de las directivas, la carencia de estímulos y la falta de interés por ejercer el liderazgo que manifestaban los docentes.

En segunda instancia, se presentó como caracterización en el desarrollo del liderazgo la promoción del liderazgo docente, donde los participantes expresaron que en la institución educativa se realizaban acciones que promovían el desarrollo del liderazgo en los docentes, contando con el apoyo necesario para desarrollar su capacidad de liderazgo.

El desarrollo del liderazgo se caracteriza como uno de los elementos que promueve el liderazgo distribuido, debido a su ideología que apunta a que este puede ser ejercido por cualquier miembro de una organización. Desde esta mirada el liderazgo distribuido, busca que las organizaciones y sus líderes formales, valoren las capacidades con las que cuenta el personal y las encaminen para formar nuevos 
líderes que aporten a los objetivos planteados por la organización (Leithwood, 2009).

\section{Resultados y análisis lista de chequeo}

A continuación se presenta el análisis del proceso de observación realizado por el investigador, el cual se orientó mediante una lista de chequeo diseñada como instrumento para recolectar información sobre los factores y características que determinan el liderazgo que se desarrolla en la institución.

Las listas de chequeo fueron diligenciadas en tres reuniones organizadas por el coordinador con la participación de los docentes, las cuales tenían como objetivo principal la organización de actividades de la institución y dar información sobre diferentes procesos que se llevaban a cabo.

Al realizar el proceso de observación y verificar los ítems planteados en el instrumento, se pudo evidenciar que existía una comunicación asertiva entre los docentes de la institución que favorece el clima laboral, lo que permitía el establecimiento de relaciones de confianza, respeto y colaboración. De igual forma, el equipo de trabajo se caracterizaba por asumir con actitud positiva los retos y responsabilidades que se distribuían por parte del coordinador, quien tenía en cuenta la especialización y habilidades de los miembros del grupo al asignar las diferentes tareas para alcanzar los objetivos planteados por la institución.

En los momentos de observación, se hizo evidente la participación de los docentes y el respeto por la opinión que se daba entre ellos al plantear sus ideas; sin embargo, estas ideas no eran respaldadas por parte de la dirección de la institución, por lo cual las decisiones que se tomaban no se llevaban a cabo a través de un consenso del equipo de trabajo, sino por las directrices que establecía el coordinador de la institución.

\section{Conclusiones}

A continuación, se presentan las conclusiones de cada una de las categorías abordadas en la investigación y sus recomendaciones, las cuales dan cuenta del logro de los objetivos planteados:

- Identificar la percepción que tienen los docentes y el coordinador de la institución educativa del liderazgo y el concepto de líder.

- Identificar los criterios de referencia que se presentan en el liderazgo de los docentes de la institución.

- Proponer recomendaciones a la institución que le permitan el desarrollo del liderazgo distribuido en el grupo de docentes.

\section{Tipología de liderazgo}

En relación con esta categoría se puede determinar, que no existe claridad en el equipo de trabajo sobre el tipo de liderazgo que se lleva a cabo en la organización, debido a que se identificó que los docentes y el coordinador de la institución no compartían una percepción sobre la tipología de liderazgo que se desarrolla dentro de la misma.

Al analizar los resultados se pudo observar en las apreciaciones de los docentes el liderazgo visionario como el que presenta una mayor sintonía entre los mismos; este se ve caracterizado en la institución porque los docentes buscan líderes que poseen una mirada clara del trabajo por realizar, la transmiten y de esta forma orientan a los demás al desarrollar las diferentes labores que demanda la institución. Sin embargo, al liderazgo visionario lo siguieron ocho tipologías más, que mostraron las múltiples caracterizaciones que se tienen sobre el liderazgo y el concepto de líder entre los docentes. De igual forma, al comparar estos resultados con las respuestas dadas por el coordinador, se observó que los mismos no coincidían con los de sus seguidores, debido a que según 
él, la tipología de liderazgo que se percibe en la institución es distribuida, porque el trabajo que se realiza se desarrolla de manera compartida y todo el equipo aporta en la planeación, toma de decisiones y la resolución de los problemas que se presentan.

Teniendo en cuenta lo anterior, se puede determinar que en la institución no existe un estilo de liderazgo que oriente su proceso de gestión, por lo cual los miembros que hacen parte del establecimiento educativo adoptan un estilo guiados por su criterio, impidiendo el desarrollo acertado de las tareas que fundamentan la labor del liderazgo en los procesos que se llevaban a cabo.

\section{Trabajo en equipo}

Este aspecto, según los docentes y el coordinador, se fundamenta en el compartir de conocimientos, experiencias y emociones por parte de los docentes, acompañado de otros aspectos como la colaboración y el compromiso y la participación e interacción entre docentes y el coordinador. Estas características son compartidas por la visión que presenta Leitwood (1996) sobre el liderazgo distribuido, quien afirma que estos factores favorecen el desempeño del grupo permitiendo encaminar sus acciones al logro de los objetivos planteados.

Sin embargo, se presentan aspectos como la ausencia de espacios para la reflexión, capacitación e innovación, que no permiten a los docentes ampliar sus horizontes y apropiarse de nuevos elementos y herramientas que fortalezcan sus habilidades y aporten de forma más significativa en el grupo de trabajo; al igual que la falta de apoyo del coordinador y la desconfianza, los cuales se presentan como aspectos que pueden generar discrepancias entre los miembros del grupo, entorpeciendo la labor del trabajo en equipo y el desarrollo óptimo para obtener mejores resultados.

\section{Distribución de roles}

Esta se evidencia en la institución en aspectos relacionados con la coordinación de labores, tareas y demás actividades que ejercen los miembros del equipo de trabajo. No obstante, existe una imposición por parte del coordinador, lo cual restringe la participación activa de los miembros del equipo, impidiendo entre otras cosas, identificar nuevos líderes dentro del grupo de trabajo; situación antagónica a los planteamientos de Spillane (2006), quien sostiene que el liderazgo distribuido promueve la integración de los individuos y su trabajo para la consecución de un objetivo común, lo que demanda que los sujetos que pertenecen al equipo de trabajo adquieran diversos roles y responsabilidades, las cuales deben ser coordinadas entre los mismos y los líderes formales, lo que conlleva que la práctica del liderazgo se desarrolle de forma compartida, encontrándose más líderes de los que se logran identificar en la organización, y el liderazgo adquiera forma de red donde el mismo se distribuye.

No obstante, la distribución de roles que se realiza dentro de la institución cumple con las demandas requeridas y es funcional lo que promueve una construcción social del liderazgo, que es lo que plantea la tipología distribuida que favorece la tarea de liderar mediante las interacciones que se logran establecer entre las personas que hacen parte de una organización.

\section{Toma de decisiones}

Se puede establecer que la toma de decisiones en la institución se da de forma autoritaria y subordinada, ya que las personas que pertenecen al equipo de trabajo no cuentan con autonomía y se les impide participar de la toma de decisiones, debido a que estas están dirigidas desde la dirección, en este caso el coordinador de la sede. Teniendo en cuenta lo anterior, la toma de decisiones que se desarrolla en la institución reprime la integración y participación de las personas que están direc- 
tamente involucradas en los procesos y en la consecución de acuerdos que se llevan en la misma, debido a que prevalece el autoritarismo por parte del directivo a cargo, evitando que las personas que conforman el equipo de trabajo compartan sus puntos de vista en pro de la consecución de acuerdos, búsqueda de soluciones y toma de decisiones que corresponden a la institución. Este aspecto está en contraposición de lo planteado por Heck y Hallinger (2005), quienes destacan que el liderazgo debe construirse como un proceso que permita descentralizar la gestión, mediante la integración de la comunidad educativa (padres, directivos, estudiantes y docentes) en las decisiones que competen a la institución, debido a que son estas personas quienes conocen su contexto y las necesidades del mismo, lo que los llevará a asumir de forma responsable y ecuánime la gestión de la institución.

Teniendo en cuenta el planteamiento anterior la caracterización de la toma de decisiones en la institución no da lugar a que se desarrolle uno de los elementos que prima en el liderazgo distribuido (la toma de decisiones compartida) y, por ende, el desarrollo del mismo en la organización.

\section{Desarrollo del liderazgo}

En relación con esta categoría se concluye, que los docentes y el coordinador coinciden en la ausencia de liderazgo docente, los primeros apuntan a que este factor se da por la falta de oportunidades y estímulos para ejercer este tipo de labor por parte de las directivas; mientras el coordinador sostiene que este aspecto se refleja por la resistencia al cambio que presentan los docentes antiguos, por reflejar desinterés, apatía y falta de compromiso ante el liderazgo. Esta situación no comparte los postulados de Leithwood (2009), quien plantea que las personas que están encargadas de dirigir, tienen la labor de coordinar las tareas y responsabilidades que asignan, con el objetivo de promover el liderazgo en los seguidores, potencializando las capacidades y habilidades en los mismos, haciendo un seguimiento y retroalimentación de sus acciones cuando se requiera; esto con el objetivo de generar un liderazgo que se distribuya en toda la organización. De acuerdo con este planteamiento, la falta de promoción del liderazgo, por parte de la coordinación, impide el surgimiento de nuevos líderes y el desarrollo de nuevas estrategias en la gestión de la institución, imposibilitando que se lleve a cabo un liderazgo distribuido.

Por otra parte, la actitud renuente al cambio y la no acogida a nuevas propuestas que según el coordinador muestran los docentes antiguos, se convierten en un obstáculo para el desarrollo de un nuevo estilo de liderazgo como lo es el distribuido, en el que los maestros como agentes protagonistas de los procesos educativos son partícipes y generadores de las transformaciones que requieren los establecimientos educativos y en la forma como estos se gestionan, por lo cual, son ellos quienes se convierten en las personas idóneas para intervenir en los procesos, adquiriendo un compromiso ante el cambio, capacitándose constantemente para establecer las mejoras educativas necesarias. Por lo anterior, la actitud de los docentes antiguos es un factor que está en contraposición a lo que busca la institución, impidiendo que se generen nuevas propuestas que permitan desarrollar otros tipos de liderazgo.

Lo abordado en cada una de las categorías analizadas en el marco de la investigación, permite concluir que en la institución no se presente el liderazgo distribuido, debido al incumpliendo de los criterios de referencia básicos que definen esta tipología como lo son la democracia y la participación en la toma de decisiones, la distribución de roles de común acuerdo y la promoción del liderazgo por parte del coordinador y los docentes. Estos criterios sumados a otros aspectos se convierten en factores fundamentales para establecer la presencia de esta tipología de liderazgo en la institución, por lo cual al no reflejarse en el trabajo que se 
realiza dentro de la misma se concluye que el liderazgo distribuido no se hace presente.

Si bien es cierto que se logran percibir algunos elementos característicos del liderazgo distribuido, como el trabajo en equipo, basado en la colaboración, compromiso y el intercambio de conocimientos entre los participantes y la coordinación de roles, esta última impuesta por el coordinador que permite el cumplimiento y el logro de los requerimientos que demanda la institución, pero que termina afectando el clima laboral; estos factores no son suficientes para que se desarrolle esta tipología de liderazgo en la institución, pero pueden constituirse en el punto de partida para generar un cambio en la forma como se desarrolla el mismo en la organización

De esta manera es importante resaltar que para caracterizar el liderazgo distribuido en la institución este debe dar cuenta de los factores claves que lo identifican y de su aplicación y desarrollo, lo cual no se presentó al analizar los resultados de la investigación.

\section{Referencias}

Ander-Egg, E. (2001). El trabajo en equipo. Editorial Progreso.

Celis, S. C., \& Sánchez, N. J. (2014). El liderazgo distribuido en docentes de una institución educativa escolar particular.

Goleman, D. (2005). Liderazgo que obtiene resultados. Harvard Business Review, 11, 109-122.

Greenwood, W. T. (1978). Teoría de decisiones y sistemas de información: introducción a la toma de decisiones administrativas. Trillas.

Gronn, P. (2002). Distributed leadership as a unit of analysis. Leadership Quarterly, 13(4), 423-51.

Heck, R. H., \& Hallinger, P. (2005). The Study of Educational Leadership and Management Where Does the Field Stand Today? Educational Management Administration \& Leadership, 33(2), 229-244.

Hernández, R, Fernández, C, Baptista, P. (2014). Metodología de la investigación.

Leithwood, K. (1996). Study: Site Management has no Effect on Scores. Education Week, vol. 16.

Leithwood, K., Mascall, B., \& Strauss, T. (2009). New perspectives on an old idea. In Distributed leadership according to the evidence, 1-14.

López, J., Sánchez, M. R., \& Altopiedi, M. (2011). Comunidades profesionales de práctica que logran sostener procesos de mejora institucional en las escuelas. Revista de Educación, 356, 109-131.

Spillane, J. P, Halverson, R., y Diamond, J. (2004). Theory of leadership practice: a distributed perspective. Journal of Curriculum Studies, 36(1) 3-34.

Spillane, J. (2006). A Distributed Perspective on Leadership: Essential Elements. Distributed Leadership, 1, 11-12. 